\title{
Surgical Societies Must Lead the Way on Addressing Bias and Microaggressions
}

\author{
Callisia N. Clarke, MD, MS \\ Division of Surgical Oncology, Medical College of Wisconsin, Milwaukee, WI
}

Bias can be defined as a systematic error introduced into sampling or testing by selecting or encouraging one outcome over others. ${ }^{1}$ As surgeons, we strive daily to mitigate the effects of bias on patient care through the routine use of evidence-based practice. As researchers we make special considerations during study design, data collection and analysis to mitigate inherent biases on study outcomes and conclusions. By no means, does this mean we have eliminated bias as a profession, in fact the opposite is true. However, the normalization of discussions around bias as a systematic problem in health care delivery and clinical research, has resulted in significant improvements as we aim to close the health care gap, and improve generalizability and interpretation of clinical trial results.

Unfortunately, conversations around mistreatment within the profession of surgery are far less common and are still emotionally fraught. This is because, the act of exacting bias during interpersonal interactions is still largely viewed as an individual or personal problem, generating feelings of anger, emotional distress, and tension when these incidents are recognized. While we have made significant progress in addressing overt or explicit bias, other forms of bias remain rampant within the profession and our surgical societies. However, while some forms of bias are due to conscious, discriminatory personal beliefs (explicit bias), the vast majority occur below our level of consciousness and are reinforced within organizations and societies without intent (unconscious and

(C) Society of Surgical Oncology 2021

First Received: 7 October 2021

Accepted: 12 October 2021;

Published Online: 2 November 2021

C. N. Clarke, MD, MS

e-mail: cnclarke@mcw.edu implicit bias). These beliefs and stereotypes manifest as microassaults, microinsults and microinvalidations; collectively referred to as microaggressions (Table 1). The cumulative effects of bias and microaggressions are evidenced in the lack of diversity and inclusion in surgery and surgical leadership. Over the last three decades, we have seen a steady increase in female representation within surgery with an approximate 3 -fold increase in the number for female surgical faculty. Female residents now account for approximately $40 \%$ of all general surgery trainees. ${ }^{2}$ Still, women account for only $20 \%$ of associate professors of surgery, $10 \%$ of full professors of surgery and remain significantly under-represented in surgical leadership., Low racial and ethnic diversity in surgery is persistent at all levels of surgical training and practice, with very little progress on the horizon. In 2020, only 5.3\% of general surgery residents identified as Black, $8.1 \%$ as Hispanic/ Latino, 0.8\% American Indian and 0.3\% Pacific Islander. ${ }^{5,6}$ While individuals certainly have a personal and professional duty to address bias, organizations and surgical societies must also strive to minimize the effects of bias, by creating an atmosphere of equity, inclusivity, tolerance, and empowerment in order to retain members and attract individuals from diverse backgrounds.

Inconsistencies in use of professional titles when introducing colleagues and trainees in the workplace and at professional meetings has long been a subtle but damaging microinvalidation that has been pervasive throughout the medical profession. Professional titles such as "Doctor" or 'Professor" convey personal expertise and authority. Consequently, the exclusion of these salutations may imply professional inferiority. Several studies have evaluated disparities in the use of professional titles by gender, race and ethnicity, as well as academic rank when introducing speakers. Files et al. evaluated the use of professional titles at 124 consecutive internal medicine grand rounds and 
TABLE 1 Definition of the types of bias and microaggressions

\begin{tabular}{|c|c|}
\hline Term & Definition \\
\hline Explicit bias & $\begin{array}{l}\text { Conscious, intentional, negative beliefs, judgments, and stereotypes which can be measured by self-report. }{ }^{13} \text { Examples } \\
\text { include, racism and misogyny. }\end{array}$ \\
\hline Unconscious bias & $\begin{array}{l}\text { Deeply engrained beliefs and learned stereotypes that automatic and unintentional. These biases occur below the level of } \\
\text { consciousness and occur naturally as we categorize experiences. These are biases based on past experience and may not } \\
\text { be rooted in ill-will. }{ }^{14}\end{array}$ \\
\hline Implicit Bias & $\begin{array}{l}\text { A form of unconscious bias that drives discriminatory behavior. Actions or judgments that are under the control of } \\
\text { automatically activated evaluation, without the performer's awareness of that causation. Occurs without conscious } \\
\text { intention and is not self-reported. }\end{array}$ \\
\hline Microassaults & Conscious and intentional discriminatory behaviors that aim to insult or belittle marginalized groups ${ }^{16}$ \\
\hline Microinsults & $\begin{array}{l}\text { Subtle verbal or nonverbal communications that are rude and insensitive and demean an individual's gender, racial, } \\
\text { religious, or sexual identity. }\end{array}$ \\
\hline Microinvalidations & Subtly exclude, negate, or nullify the experiences of marginalized groups ${ }^{16}$ \\
\hline
\end{tabular}

reported that males were less likely to use professional titles when introducing speakers, when compared to female introducers $(65.6 \% \quad[141 / 215] \quad$ vs. 96.2\% [102/106]; $p<0.001)^{7}$ Female speakers were less likely to be introduced using professional titles and this difference was almost exclusively found when female speakers were introduced by males $49.2 \%$ (31/63) versus female $97.8 \%$ (45/46) $(p<0.001) .^{7}$ Duma et al. found similar results in their study of the use of professional titles at the American Society of Clinical Oncology (ASCO) Annual Meeting. ${ }^{8}$ Female speakers were less frequently addressed using professional titles when compared to their male counterparts $(62 \% v 81 \% ; p<.001) .^{8}$ Males were less likely to use professional address when introducing female speakers compared to when introducing male speakers $(53 \% v 80 \% ; p<0.01)$. There was no difference in the use of professional address when introducers were female. ${ }^{8}$ These studies give limited insight into the ubiquitous nature of microinsults within organizations and medical societies. They are flawed by the binary assignment of sex, based in some cases only by gender expression, though efforts were made to identify preferred pronouns when available. Additionally, the paucity of racial and ethnic minorities invited into these exclusive spaces could not allow for meaningful comparisons, a topic for another day.

The gender gap in leadership of surgical societies persists despite increased female representation in the field. A recent analysis of gender parity in general surgical societies noted $83 \%$ male representation amongst society leaders when compared to only $17 \%$ female. ${ }^{9}$ Others studies show that these disparities are most pronounced at the highest echelons of leadership, with the largest gender gap seen at the level of society president. ${ }^{10}$ For these reasons, it is especially important that the Society of Surgical Oncology (SSO) and other surgical societies lead efforts to reduce bias and microaggression within the organizational leadership and at all society authorized meetings and initiatives.

An analysis of the 2018 and 2019 SSO Annual Meetings found relative parity in the proportion of female speakers $(42 \%)$ with no difference by gender in the use of professional titles when introducing speakers. ${ }^{11}$ However, residents and fellows were less likely to be introduced using professional titles with an odds ratio of 2.77 (1.41 5.42) $p=0.003 .^{11}$ Unfortunately, the effect of race and ethnicity in this study could not be access given the paucity of assigned Black (1\%) and Hispanic (3.4\%) speakers identified by perceived race. ${ }^{11}$ These data inspired the development and implementation the SSO Guidelines for Respectful Discourse which was dispersed to all meeting leaders and moderators. ${ }^{12}$ These guidelines, in short, encouraged consistent forms of professional address during speaker introductions, the use of gender-neutral language when applicable, and patient centered language when discussing disease and/or treatment effects. Implementation of these guidelines resulted in an increase in professional titles used during speaker introductions from $70 \%$ in 2018 and 2019 to $84 \%$ in the 2021 SSO Annual Meeting. ${ }^{12}$ The authors and the SSO should be congratulated on these efforts which underscore that when treated as a systematic problem, implicit bias and microaggressions can be mitigated with measurement and acknowledgement of organizational blind spots and data-driven development and implementation of effective interventions.

In order to endure, surgical societies including the SSO must strive to encourage new member enrollment, engagement and retention. At the core of these efforts is the need to create diverse and inclusive spaces, maintain youth, vibrancy and relevance given the ever-changing demographics of the surgical workforce. Surgical societies are uniquely qualified to lead efforts to ameliorate the toxic 
effects of bias and microaggression in surgery as we strive to create a diverse physician workforce that reflects the demographics of the patient populations we serve and reduce disparities in surgical outcomes. I believe, there are several steps are critical to successful DEI efforts, some of which are briefly outlined below.

\section{Diversity, equity, inclusion (DEI) and justice must be} a central pillar of the organization's mission. In doing so DEI efforts should be wove throughout all aspects of the organizational structure and initiatives; just as critical to the success of the organization as other stated pillars. Societies should write a statement that clearly states why DEI efforts are critical to the organizations' success and make that statement public.

2. Define the problem. An in-depth assessment of the organization's membership, leadership and culture should be undertaken to identity and prioritize targeted areas for improvement. Surveys to assess member perception of organizational culture and objective data to evaluate the membership demographics are usually necessary to guide effective programs. Define what DEI means to your organization and remember to look at variables (outside of sex, gender identity, sexual orientation, race, ethnicity, disability, and religion to name a few) not often measured, that may be relevant to your society or specialty. For example, diversity in institutional representation, or diversity in leadership age or academic rank may be a critical area to address in some organizations.

3. Establish and empower a committee or advisory board to generate a strategic plan to address target areas identified. Ensure that the committee directly reports to organizational leadership to maximize efficiency. Start with a combination of short term and long-term goals to ensure continued momentum. The strategic plan must be re-evaluated at regular intervals and adjusted as needed. Adequate finances and additional resources should be provided to safeguard development and execution of the strategic plan.

4. Implement data-driven interventions. Measure the effectiveness and impact of these interventions objectively whenever possible. Stewart et al. ${ }^{12}$ exemplify this in the recent manuscript.

5. Competency-based assessment of leadership and expertise with a particular focus on DEI should be the guiding principle that determines suitability for meeting and society leadership and participation. Ensure a transparent process for election to leadership roles and mostly importantly, actively work to eliminate reliance on "known networks" as a method of identifying talent. In general, casting a wider net allows for more inclusive recruitment and a larger, more diverse talent pool to choose from.

\section{REFERENCES}

1. https://www.merriam-webster.com/dictionary/bias. October 5, 2021].

2. Hu Y-Y, et al. Discrimination, Abuse, Harassment, and Burnout in Surgical Residency Training. New England Journal of Medicine. 2019;381(18):1741-52.

3. Hu YY, et al. Discrimination, Abuse, Harassment, and Burnout in Surgical Residency Training. $N$ Engl $J$ Med. 2019;381(18):1741-52.

4. Abelson JS, et al. The climb to break the glass ceiling in surgery: trends in women progressing from medical school to surgical training and academic leadership from 1994 to 2015. Am J Surg. 2016;212(4):566-572.e1.

5. Association of American Medical Colleges (2020). Report on Residents. Retrieved from [https://www.aamc.org/data-reports/st udents-residents/interactive-data/report-residents/2020/table-b5md-residents-race-ethnicity-and-specialty]. Accessed 10/1/2021.

6. Association of American Medical Colleges (2019). Diversity in Medicine: Facts and Figures 2019. Retrieved from [https://ww w.aamc.org/data-reports/workforce/interactive-data/figure-18-per centage-all-active-physicians-race/ethnicity-2018]. Accessed $10 / 1 / 2021$

7. Files JA, et al. Speaker introductions at internal medicine grand rounds: forms of address reveal gender bias. Journal of women's health. 2017;26(5):413-9.

8. Duma N, et al. Evaluating Unconscious Bias: Speaker Introductions at an International Oncology Conference. Journal of Clinical Oncology. 2019;37(36):3538-45.

9. Wu, B., et al., Gender disparity in leadership positions of general surgical societies in North America, Europe, and Oceania. Cureus, 2019. 11(12).

10. Lyons NB, et al. Gender disparity in surgery: an evaluation of surgical societies. Surgical infections. 2019;20(5):406-10.

11. Stewart CL, et al. Unconscious Bias in Speaker Introductions at a Surgical Oncology Meeting: Hierarchy Reigns Over Gender. Annals of Surgical Oncology. 2020;27(10):3754-61.

12. Stewart, C.L., et al., Data-Driven Respectful Discourse in the Society of Surgical Oncology. Annals of Surgical Oncology, 2021.

13. Boysen GA. A review of experimental studies of explicit and implicit bias among counselors. Journal of Multicultural Counseling and Development. 2009;37(4):240-9.

14. Fiarman SE. Unconscious bias: When good intentions aren't enough. Educational Leadership. 2016;74(3):10-5.

15. Greenwald AG, Banaji MR. Implicit social cognition: attitudes, self-esteem, and stereotypes. Psychol Rev. 1995;102(1):4-27.

16. Sue DW, et al. Racial microaggressions in everyday life: implications for clinical practice. Am Psychol. 2007;62(4):271-86.

Publisher's Note Springer Nature remains neutral with regard to jurisdictional claims in published maps and institutional affiliations. 